\title{
Etiology and Antimicrobial Susceptibility Profile of Isolates from Ascitic Fluid of Patients with Spontaneous Bacterial Peritonitis
}

\author{
Priyam Chawla ${ }^{1}$, Deepinder Kaur ${ }^{1}$, Rajoo Singh Chhina ${ }^{2}$, Veenu Gupta ${ }^{1}$, \\ Jyoti Chaudhary', Manisha Aggarwal ${ }^{1}$ \\ Department of Microbiology ${ }^{l}$, Department of Gastroenterology ${ }^{2}$ \\ Dayanand Medical College \& Hospital, Ludhiana.
}

\begin{abstract}
Background \& Objectives: Ascitic fluid infections are frequent complications among patients of cirrhosis with ascities, of which spontaneous bacterial peritonitis (SBP) is the most common and potentially fatal. This study was planned to know the etiology and current antibiotic susceptibility profile of the isolates from the SBP patients.

Material and Methods: A total of 50 cases of SBP from cirrhosis patients with ascites were enrolled in this study. Samples were processed in automated Bactec or Bac-T/Alert. Further identification \& antimicrobial susceptibility testing was done by VITEK-2 system.

Results: Most common organism isolated was Escherichia coli (40\%) followed by Coagulase negative Staphylococci, Klebsiella pneumonia and Acinetobacter baumanii. Among all Gram negative isolates, $94.7 \%$ were sensitive to tigecycline, $92.1 \%$ sensitive to colistin. It also showed high susceptibility to amikacin and carbapenems while low susceptibility was seen to others. All the gram positive organisms were sensitive to vancomycin \& linezolid and show moderate sensitivity to ciprofloxacin, tetracycline, cotrimoxazole, and gentamycin. Low sensitivity was seen to penicillin. Methicillin resistant coagulase negative Staphylococci (MRCoNS) were seen in 4 (57\%) isolates.

Interpretation \& Conclusion: Escherichia coli is the most common cause of SBP. Antimicrobial resistance is increasing therefore early detection and determination of antimicrobial susceptibility pattern is important to reduce the mortality and morbidity associated with ascitic fluid infections.
\end{abstract}

Keywords: Spontaneous bacterial peritonitis, ascitic fluid

\section{INTRODUCTION}

Ascites is the most common complication of cirrhosis of liver and indicate the presence of liver degeneration \& hepatic decompensation. ${ }^{1}$ Patients with cirrhosis have increased risk of developing bacterial infections. Ascitic fluid infections are a frequent complication among patients of cirrhosis with ascites. Ascitic fluid infection

\section{Corresponding Author :}

Dr. Deepinder Kaur,

Professor \& Head,

Department of Microbiology,

Dayanand Medical College \& Hospital, Ludhiana

Mobile No.: 9872890032

Email : deepiderchhina@rediffmail.com can be classified into five categories based on ascitic fluid culture results, polymorphonuclear (PMN) cell counts and presence or absence of surgical cause of infection. The variants of spontaneous bacterial peritonitis (SBP) are classical SBP, culture-negative neutrocytic ascites(CNNA), monomicrobial nonneutrocytic bacterascites (MNB). ${ }^{2}$

Among ascitic fluid infections, $\mathrm{SBP}$ is the most common $\&$ one of the leading cause of morbidity \& mortality. It is defined as monomicrobial infection of ascitic fluid, which is not accompanied by an intra abdominal surgical source of infection. The diagnosis of SBP is established by an elevated ascitic fluid PMN count ( $\geq 250$ cells/ $\mathrm{mm}^{3}$ ) and a positive ascitic fluid culture. ${ }^{3,4}$ Prevalence of SBP in cirrhotic patients varies in different parts of the world. In India it ranges from 9.3\%- 34.92\%. Early 
detection and identification of clinically relevant microorganisms in ascitic fluid culture and determination of antimicrobial susceptibility pattern for appropriate administration of antimicrobial therapy has been shown to reduce mortality and morbidity associated with ascitic fluid infection. Therefore, the present study was undertaken to know the microbiological profile and current antibiotic susceptibility patterns of isolates from patients with SBP admitted in Gastroenterology ward and ICU in a teaching hospital in North India.

\section{MATERIAL \& METHODS}

A total of 50 cases of SBP from cirrhosis patients with ascities (diagnosed on the basis of PMN $>250 / \mathrm{mm}^{3} \&$ Culture positive) were enrolled in study. Ascitic fluid samples were processed in Bactec or Bac-T/Alert microbial detection system. Smears were prepared from the positive culture bottles and Gram staining of the smears was done. Simultaneously all the positive bottles were sub cultured on blood agar and MacConkey's agar plates. The plates were incubated at $37^{\circ} \mathrm{C}$ for $18-24$ hours. Identification of isolated organisms and antimicrobial susceptibility was done by VITEK-2 system. ${ }^{5}$

\section{RESULTS}

Among 50 SBP enrolled cases majority were male patients $(86 \%)$ and most common risk factor was alcohol consumption (70\%) followed by viral hepatitis. Mean age of the patients was 52.4+/-11.6 years. Out of 50 isolates, majority were Gram negative (76\%), followed by Gram positive (20\%) and yeasts (4\%). Among Gram negative isolates most common was Escherichia coli (E.coli) (40\%), followed by Klebsiella pneumoniae ( $K$. pneumonia) (14\%) \& Acinetobacter baumanii (12\%). Most common Gram positive isolates were coagulase negative Staphylococci (CoNS) (14\%) followed by Enterococcus faecium (6\%). (Figure I) Among all Gram negative isolates, $94.7 \%$ were sensitive to tigecycline, $92.1 \%$ sensitive to colistin. It also showed high susceptibility to amikacin (73.7\%), carbapenems (57.8$71 \%$ ), while moderate susceptibility was seen to cefoperazone/ sulbactam (50\%), piperacillin/ tazobactam (50\%), cotrimoxazole (47.3\%), fluoroquinolones $(31.5 \%)$ and low susceptibility to cefepime (28.9\%) and amoxicillin/ clavulanic acid
(20\%) was observed. (Figure II) All the gram positive organisms were sensitive to vancomycin \& linezolid and showed moderate sensitivity to tetracycline $(50 \%)$, ciprofloxacin $(50 \%)$, cotrimoxazole $(42 \%)$ and gentamycin $(40 \%)$. Low sensitivity was seen to penicillin (30\%). Methicillin resistant coagulase negative Staphylococci (MRCONS) were seen in $4(57 \%)$ isolates. Yeast cells $(4 \%)$ were identified as Candida albicans and were sensitive to fluconazole, voriconazole, flucytocytosine, amphotricine B and capsofungin drugs. Out of the total SBP cases, seven $(14 \%)$ patients were expired.

\section{DISCUSSION}

Bacterial infections are very common in patients with cirrhosis and currently represent one of the most important reasons for progression of liver failure. In recent years, infections caused by multi-drug resistant bacteria are increasing at an alarming rate thus early identification and management of infection with appropriate antibiotics is very important to reduce the

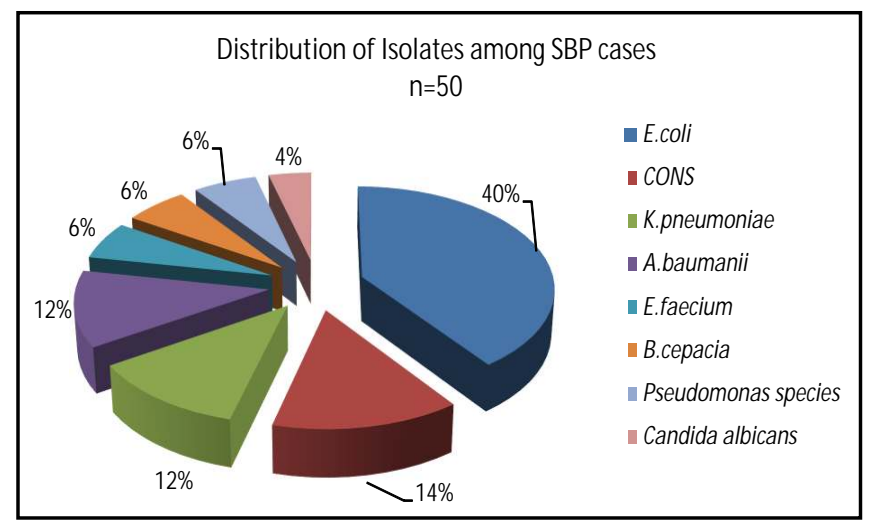

Fig. I: Distribution of Isolates among SBP cases $n=50$

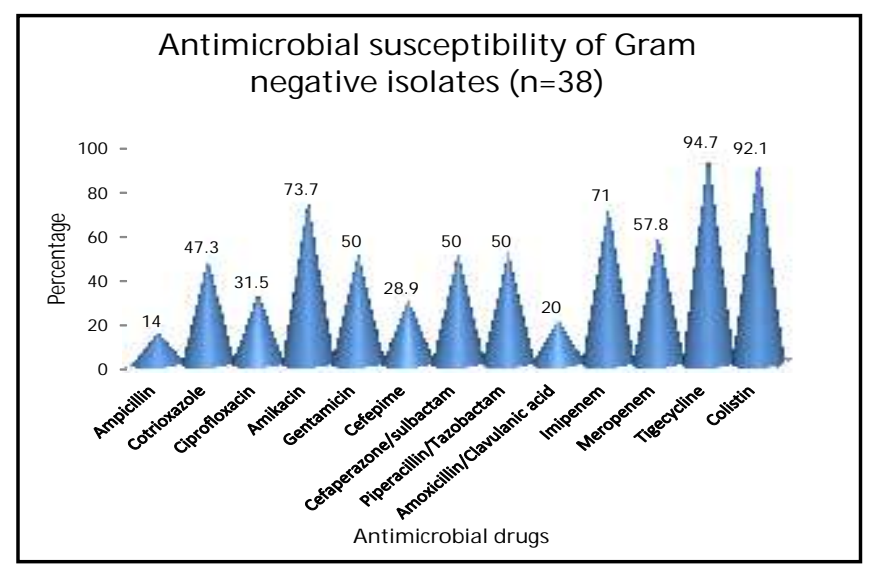

Fig. II: Antimicrobial susceptibility of Gram negative isolates $(n=38)$ 
disease burden.

In the present study majority of the cases were males $(86 \%)$. High male to female ratio was seen in this study (6.1:1) as alcohol consumption is more common among males in our country which is the most common cause of cirrhosis. A similar male to female ratio (8:1) has also been reported in literature. ${ }^{6}$ In our study the mean age of the patient suffering from cirrhosis is 52.4 years which is nearly correlating with the mean age in various studies (51-54 years). ${ }^{7.8}$

We found that, majority of patients had history of alcoholism (70\%) as the underlying cause of cirrhosis followed by viral hepatitis. This is consistent with other study. ${ }^{6}$ Whereas some authors gave contrasting results, they reported hepatitis $\mathrm{C}$ as commonest underlying cause of cirrhosis in 139 (74.3\%) followed hepatitis B infection in 43 (22.9\%) patients. $^{9}$

Results in our study depict that Gram negative organisms were found as the more common (76\%) cause of SBP than Gram positive organisms (20\%). Fungal infections were not found common in our study. Among the Gram negative isolates E.coli was most common isolate followed by K.pneumoniae and A.baumanii. Similarly in several other studies E.coli was found the most common cause of ascitic fluid infection (30\%$75 \%)^{10,11}$

In the current study antimicrobial susceptibility pattern among the gram negative organisms showed high susceptibility to tigecycline $(94.7 \%)$ and colistin (92.1\%) along with amikacin $(73.7 \%)$ and carbapenems (58-71\%) whereas another study showed fairly high susceptibility to amikacin $(92.3 \%){ }^{12}$ However low susceptibility was seen to cefepime (29\%), amoxicillin/ clavulanic acid (20\%) which was comparable to results in other studies done in Egypt and Mumbai.12,13 Ciprofloxacin susceptibility was seen in $31.5 \%$ of Gram negative isolates, whereas slightly lower percentage was observed in other studies $(17.71 \%, 20 \%, 20 \%)$. $^{12-14}$

Among the Gram positive isolates majority were CONS (70\%) followed by E.faecium (30\%). Gram positive organisms in the current study showed highest susceptibility to linezolid, vancomycin and moderate to flouroquinolones, tetracyclines and aminoglycosides and and low sensitivity to penicillin. These results correlated with a study done in Tehran. ${ }^{15}$ All Enterococcal isolates were susceptible to vancomycin. Methicillin resistance was seen in $57 \%$ of CoNS and which was quite high compared to another study where low MRCoNS (16.7\%) were reported. These epidemiological changes may be attributed to the long term antibiotic therapy as primary or secondary prophylaxis in high risk cirrhotic patients to reduce recurrence and improve survival of SBP patients. 13 Out of the 50 cases of SBP, 7(14\%) patients expired which was similar to mortality rate seen in another study $(8.5 \%$, $12.2 \%){ }^{16,9}$

Bacteria causing SBP are recently showing changes in the etiological profile and multi-drug resistant opportunistic pathogens emerging as causal agents of SBP together with an increase in resistance to antibiotics commonly used for the empiric treatment of SBP due to inappropriate use of broad spectrum antibiotics. Preventive measures like continuous surveillance of the wards and ICUs and a strict implementation of infection control practice can go a long way in containing the menace of drug resistance in the heath care settings.

\section{References}

1. Bacon BR. Cirrhosis and its complications. In: Longo DL, Fauci AS, Karper DL, Hausen SL, Jameson JL, Loscalzo J editors. Harrison's principles of Internal medicine. 18th ed McGraw Hill New York;2012.p.2592-3.

2. Runyon BA. Ascites and spontaneous bacterial peritonitis. In: Feldman M, Friedman LS, Brandt LJ editors. Sleisenger, Fordtran's Gastrointestinal and liver disease Pathophysiology/ Diagnosis/Management. 9th ed Saunders Elsevier Philadelphia; 2012.p.1517-25.

3. European association of the study of the liver. EASL clinical practical guidelines on the management of ascites, spontaneous bacterial peritonitis, and hepatorenal syndrome in cirrhosis. Journal of Hepatology. 2010;53(3):397-417.

4. Tsao GG. Current management of the complications of cirrhosis and portal hypertension: variceal hemorrhage, ascites, and spontaneous bacterial peritonitis. Gastroenterology. 2001;120 (3):726-48.

5. Winn JW, Washington W, Allen S, Janda W, Koneman E, Procop G et al. Introduction to Microbiology: Part II: Guidelines for the collection, transport, processing, analysis and reporting of cultures from specific specimen sources. In: Koneman's color atlas and textbook of diagnostic microbiology. 6th ed. Philadelphia: Lippincot Willams and Willikins; 2006.p.103-5. 
6. Nadagouda SB, Mahesh B, S.V. Kashinakunti, M S Birader. Spontaneous bacterial peritonitis in cirrhosis of liver with ascites-a cross sectional study. Int J Biol med Res. 2013;4(2): 3143-7.

7. Syed VA, Ansari Ja, Karki P, Regmi M, Khanal B. Spontaneous Bacterial Peritonitis in cirrhotic ascites: A prospective study in a tertiary care hospital, Nepal. Kathmandu Univ Med J. 2007;5:48-59.

8. Khan Z, Khan I, Ud Din J, Subhan F, Khan B, Khan H. Frequency of spontaneous bacterial peritonitis in cirrhotic patients with ascites due to hepatitis $\mathrm{C}$ virus and efficacy of ciprofloxacin in its treatment. Gomal J Med Sci. 2009;7(2):14954.

9. Kamani L, Mumtaz K, Ahmed US, Ali AW, Jafri W. Outcomes in culture positive and culture negative ascitic fluid infections in patients with viral cirrhosis: cohort study. BMC Gastroenterology. 2008;8:59.

10. Reginato TJ, Oliveira MJ, Moreira LC, Lamanna A, Acencio MM, Antonangelo L. Characteristics of ascitic fluid from patients with suspected spontaneous bacterial peritonitis in emergency units at a tertiary hospital. Sao Paulo Med J. 2011;129(5):315-9.

11. Abd Elaal MM, Zaghloul SG, Bakr HG, Ashour MA, AbdelAziz-El-Hady H, Khalifa NA, Amr GE. Evaluation of different therapeutic approaches for spontaneous bacterial peritonitis. Arab J Gastroenterol. 2012;13:65-70.

12. S Bankar, A De, S Baveja. Study of ascitic fluid for diagnosis of spontaneous bacterial peritonitis (SBP) in adult patients with cirrhosis. IJMAS. 2014;3(1): 1-9.

13. El-Bendary MM, Abdel-Aiz M, EL-Sherbiny Walid A, Farag R, El-Gilany AH, Zaghloul Mohd HE. Spontaneous Bacterial Peritonitis: Clinico-epidemiological and microbiological study. Benha Med J. 2009;26:287-9.

14. Park MK, Lee JH, Byun YH, Lee Hle, Gwak GY, Choi MS, et al. Changes in the profiles of causative agents and antibiotic resistance rate for spontaneous bacterial peritonitis: an analysis of cultured micro-organisms in recent 12 years. Korean $\mathrm{J}$ Hepatol. 2007;13(3):370-7.

15. Sheikhbahaei S, Abdollahi A, Hafezi-Nejad N, Zare E. Patterns of antimicrobial resistance in the causative organisms of spontaneous bacterial peritonitis: A single centre, six-year experience of 1981 samples. International Journal of Heptology. 2014;2014:917856.

16. Puri AS, Puri J, Ghoshal UC, Sharma BC, Saraswat VA, Ayyagari A et al. Frequency, microbial spectrum \& outcome of Spontaneous Bacterial Peritonitis. Indian J Gastroenterol. 1996; 15(3):86- 9. 